ESAIM: PROCEEDINGS, November 2005, Vol.15, 1-17

T. Goudon, E. Sonnendrucker \& D. Talay, Editors

DOI: $10.1051 /$ proc:2005020

\title{
AN INTRODUCTION TO FINITE VOLUME METHODS FOR HYPERBOLIC CONSERVATION LAWS
}

\author{
FRANCOIS BOUCHUT ${ }^{1}$
}

\begin{abstract}
Hydrodynamic transport problems often take the form of systems of hyperbolic conservation laws. This minicourse intends to introduce the main notions and tools for the numerical approximation of such systems by finite volumes. The notions of consistency, stability, and approximate Riemann solvers are explained in particular. The main ingredients to go to second-order and multidimension are given.
\end{abstract}

Résumé. Les problèmes de transport hydrodynamique prennent souvent la forme de systèmes de lois de conservation hyperboliques. Ce cours vise à introduire les notions principales et les outils pour l'approximation numérique de tels systèmes par des volumes finis. En particulier, les notions de consistance, stabilité et solveurs de Riemann approchés sont expliquées. On donne aussi les arguments principaux pour passer au second ordre et aux cas multi-dimensionnels.

Key-words: Systems of conservation laws - finite volumes - invariant domains - entropy inequalities approximate Riemann solver

AMS Subject Classification: 76M12, 65M06

\section{Contents}

1. Quasilinear systems, conservation laws 2

1.1. Quasilinear systems and conservation laws 2

1.2. Examples of conservation laws 2

1.3. Entropy 3

1.4. Entropy: examples 3

1.5. Invariant domains 3

1.6. Rankine Hugoniot jump relation 4

2. Finite volumes for one dimensional conservation laws 4

2.1. First-order scheme 4

2.2. Consistency 5

2.3. Stability 5

3. Approximate Riemann solvers 6

3.1. Riemann problem 6

3.2. Approximate Riemann solvers 6

$\begin{array}{lll}3.3 . & \text { Interpretation of an approximate solver } & 7\end{array}$

3.4. Entropy

3.5. Invariant domains $\quad 8$

${ }^{1}$ Département de Mathématiques et Applications, DMA - UMR 8553, Ecole Normale Supérieure 45, rue d'Ulm 75230 Paris cedex 05 - France. E-mail:E-mail : Francois.Bouchut@ens.fr 
3.6. Simple solvers $\quad 9$

3.7. HLL solver 10

4. Second-order schemes 10

4.1. Reconstruction operator $\quad 10$

4.2. Accuracy 11

4.3. Stability 11

4.4. Examples of reconstruction operators 11

4.5. Second-order accuracy in time 13

4.6. Test 1: Burgers equation 13

4.7. Test 2: Gas dynamics 13

5. Multidimensional problems 14

5.1. Bidimensional systems of conservation laws 14

5.2. Schemes by interface $\quad 15$

5.3. Second-order accuracy 16

5.4. Test 3: Gas dynamics, random velocity 16

$\begin{array}{ll}\text { Bibliography } & 18\end{array}$

\section{QUASILINEAR SYSTEMS, CONSERVATION LAWS}

\subsection{Quasilinear systems and conservation laws}

We recall here very basic definitions. The reader is refered to [6] for more theoretical results.

- Quasilinear system of partial differential equations (one dimension):

$$
\partial_{t} U+A(U) \partial_{x} U=0, \quad t>0, x \in \mathbb{R}
$$

where $U(t, x) \in \mathbb{R}^{p}$, and $A(U)$ is a $p \times p$ matrix.

The system is said hyperbolic if $A(U)$ is diagonalizable for all $U$.

Such system undergoes the propagation of some waves, like in $U=\varphi(x-t a), a \in \mathbb{R}$, which is solution to $\partial_{t} U+a \partial_{x} U=0$.

- System of conservation laws:

$$
\partial_{t} U+\partial_{x} F(U)=0,
$$

where $F(U) \in \mathbb{R}^{p}$. It is of the form (1.1) with $A(U)=F^{\prime}(U)$.

The main features for equation (1.1) are that (see [6])

$\triangleright$ the Cauchy problem has bounded, but discontinuous, solutions $U$,

$\triangleright$ solutions to (1.1) are not well-defined,

$\triangleright$ the nonlinearity induces nonuniqueness.

\subsection{Examples of conservation laws}

- Scalar equation

$$
\partial_{t} U+\partial_{x} F(U)=0
$$

with $U \in \mathbb{R}$ and $F: \mathbb{R} \rightarrow \mathbb{R}$. 
- The system of gas dynamics

$$
\left\{\begin{array}{l}
\partial_{t} \rho+\partial_{x} \rho u=0 \\
\partial_{t}(\rho u)+\partial_{x}\left(\rho u^{2}+p\right)=0 \\
\partial_{t}\left(\rho\left(u^{2} / 2+e\right)\right)+\partial_{x}\left(\left(\rho\left(u^{2} / 2+e\right)+p\right) u\right)=0
\end{array}\right.
$$

where $\rho \geq 0, u \in \mathbb{R}, e \geq 0$ and $p=(\gamma-1) \rho e$ with $\gamma>1$ a constant.

Here $U=\left(\rho, \rho u, \rho\left(u^{2} / 2+e\right)\right)$.

\subsection{Entropy}

The notion of entropy in hyperbolic problems is used for several purposes:

- selecting admissible solutions,

- getting a priori bounds,

- proving compactness (for mathematicians only...)

$\triangleright$ For the quasilinear system (1.1), i.e. $\partial_{t} U+A(U) \partial_{x} U=0$, an entropy is a scalar function $\eta(U)$, such that there exist another scalar function $G(U)$, called the entropy flux, satisfying

$$
G^{\prime}(U)=\eta^{\prime}(U) A(U)
$$

$\triangleright$ Interest: smooth solutions to (1.1) satisfy $\partial_{t} \eta(U)+\partial_{x} G(U)=0$.

If $\eta$ is a convex entropy, a solution $U$ to (1.1) is said $\eta$ entropy satisfying if

$$
\partial_{t} \eta(U)+\partial_{x} G(U) \leq 0
$$

Under suitable boundary conditions, it implies the a priori bound

$$
\frac{d}{d t} \int \eta(U) d x \leq 0
$$

\subsection{Entropy: examples}

- For scalar conservation laws, any convex function $\eta(U)$ is an entropy, with entropy flux defined by $G^{\prime}=\eta^{\prime} F^{\prime}$.

- For the system of gas dynamics, an entropy is given by

$$
\eta(U)=\rho \phi\left(\frac{p^{1 / \gamma}}{\rho}\right), \quad G(U)=\rho u \phi\left(\frac{p^{1 / \gamma}}{\rho}\right),
$$

with $\phi^{\prime} \leq 0$ and $\phi^{\prime \prime} \geq 0$.

In practice, in order to find an entropy one tries to derive a new conservation law by formal multiplication of the original conservation laws by nonlinear functions of $U$.

\subsection{Invariant domains}

We say that a convex set $\mathcal{U} \subset \mathbb{R}^{p}$ is an invariant domain for (1.2) if it has the property that

$$
U^{0}(x) \in \mathcal{U} \text { for all } x \Rightarrow U(t, x) \in \mathcal{U} \text { for all } x, t>0 .
$$

The convexity property is with respect to the conservative variable $U$. For the theory, refer to [6]. 
- Example: for a scalar law $(p=1)$, any closed interval is an invariant domain.

- Example: For the full gas dynamics system, the set where $e>0$ is an invariant domain (check that this set is convex with respect to the conservative variables $\left(\rho, \rho u, \rho\left(u^{2} / 2+e\right)\right)$.

The property for a scheme to preserve an invariant domain is an important issue of stability. In particular, the occurrence of negative values for density of for internal energy in gas dynamics calculations leads rapidly to breakdown in the computation.

\subsection{Rankine Hugoniot jump relation}

It characterizes weak solutions of conservation laws with a discontinuity accross a smooth curve. Consider a

FIGURE 1. Discontinuity across a smooth curve

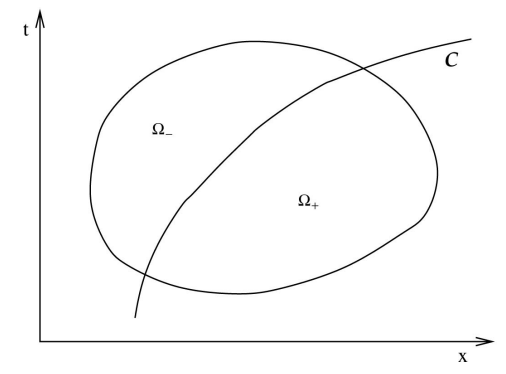

function $U(t, x)$ that is $C^{1}$ on $\bar{\Omega}_{-}$and in $\bar{\Omega}_{+}$, as shown on Figure 1. Then $U$ solves $\partial_{t} U+\partial_{x} F(U)=0$ in $\Omega$ if and only if $U$ is a classical solution in $\Omega_{-}$and $\Omega_{+}$, and the Rankine-Hugoniot jump relation

$$
F\left(U_{+}\right)-F\left(U_{-}\right)=\dot{\xi}\left(U_{+}-U_{-}\right) \quad \text { on } \mathcal{C} \cap \Omega
$$

is satisfied, where the curve $\mathcal{C}$ is parametrized by $x=\xi(t)$.

\section{Finite VOlumes FOR ONE Dimensional CONSERVATION LAWS}

\subsection{First-order scheme}

For the conservation law (1.2), i.e. $\partial_{t} U+\partial_{x} F(U)=0$, a first-order conservative finite volume scheme is a scheme of the form

$$
U_{i}^{n+1}-U_{i}+\frac{\Delta t}{\Delta x_{i}}\left(F_{i+1 / 2}-F_{i-1 / 2}\right)=0
$$

where $U_{i}^{n}$ is an approximation of the solution $U(t, x)$,

$$
U_{i}^{n} \simeq \frac{1}{\Delta x_{i}} \int_{x_{i-1 / 2}}^{x_{i+1 / 2}} U\left(t_{n}, x\right) d x
$$

the indices $n$ and $i$ refer to time $t_{n}$ and cell $C_{i}=\left(x_{i-1 / 2}, x_{i+1 / 2}\right)$ of size $\Delta x_{i}=x_{i+1 / 2}-x_{i-1 / 2}$, where $t_{n+1}-t_{n}=\Delta t$ and $\cdots<x_{i-1 / 2}<x_{i+1 / 2}<\ldots$ is the grid. The numerical fluxes $F_{i+1 / 2}, i \in \mathbb{Z}$, are defined by

$$
F_{i+1 / 2}=F\left(U_{i}, U_{i+1}\right)
$$

and $F\left(U_{l}, U_{r}\right)$, is the numerical flux function, to be chosen satisfying some precision and stability properties. 


\subsection{Consistency}

$\triangleright$ The consistency of a numerical method is the minimal precision property we can ask for: if the sequence $U_{i}^{n}$ converges to a smooth function, it must be a solution to (1.1).

$\triangleright$ For the finite volume method associated to the numerical flux $F\left(U_{l}, U_{r}\right)$, the consistency is defined as

$$
F(U, U)=F(U)
$$

(Notation: $F\left(U_{l}, U_{r}\right)$ is the numerical flux, $F(U)$ is the exact flux)

Proposition 2.1. Assume that for all $i$,

$$
U_{i}^{n}=\frac{1}{\Delta x_{i}} \int_{C_{i}} U\left(t_{n}, x\right) d x
$$

for some smooth solution $U(t, x)$ to (1.2), and define $U_{i}^{n+1}$ by (2.1)-(2.3). If the scheme is consistent, then for all $i$,

$$
U_{i}^{n+1}=\frac{1}{\Delta x_{i}} \int_{C_{i}} U\left(t_{n+1}, x\right) d x+\Delta t\left(\frac{1}{\Delta x_{i}}\left(\mathcal{F}_{i+1 / 2}-\mathcal{F}_{i-1 / 2}\right)\right),
$$

where the conservative error $\mathcal{F}_{i+1 / 2}$ verifies

$$
\mathcal{F}_{i+1 / 2} \rightarrow 0
$$

as $\Delta t$ and $\sup _{i} \Delta x_{i}$ tend to 0 .

The proof is given in [1].

\subsection{Stability}

$\triangleright$ Stability properties are usually required for a finite volume scheme. They can be of two types:

- Preservation of some invariants domains: $U_{i}^{n} \in \mathcal{U} \forall i \Rightarrow U_{i}^{n+1} \in \mathcal{U} \forall i$. This means that some natural bounds are preserved during the evolution, like nonnegativity of density.

- Existence of a discrete entropy inequality

$$
\eta\left(U_{i}^{n+1}\right)-\eta\left(U_{i}\right)+\frac{\Delta t}{\Delta x_{i}}\left(G_{i+1 / 2}-G_{i-1 / 2}\right) \leq 0
$$

for some numerical entropy flux $G_{i+1 / 2}=G\left(U_{i}, U_{i+1}\right)$. It selects suitable solutions, and provides the a priori bound $\sum_{i} \Delta x_{i} \eta\left(U_{i}^{n}\right) \leq \sum_{i} \Delta x_{i} \eta\left(U_{i}^{0}\right)$.

- A variant of this property is the existence of a semi-discrete entropy inequality, that is valid only in the limit $\Delta t \rightarrow 0$.

$\triangleright$ These stability properties can only hold under a CFL condition

$$
a_{i+1 / 2} \Delta t / \Delta x_{i} \leq 1
$$

where $a_{i+1 / 2}$ is a suitable approximation of the wave speed. This CFL condition is related to the numerical flux considered, and has to be determined by a precise analysis of it, in such a way that it implies as many stability properties as possible.

Notice that in $(2.1),(2.3)$, the value $U_{i}^{n+1}$ depends on three values at time $t_{n}: U_{i}^{n}, U_{i-1}^{n}, U_{i+1}^{n}$, thus in order to establish the entropy inequality or invariance property, one has merely three independent variables.

It is possible to reduce the problem to two variables $U_{i}^{n}, U_{i+1}^{n}$, and therefore to study an interface $i+1 / 2$ problem. The most natural way to see this is via an approximate Riemann solver. 


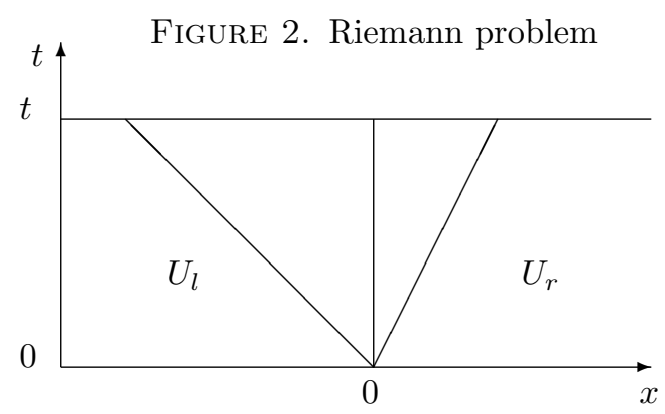

\section{Approximate Riemann solvers}

\subsection{Riemann problem}

The Riemann problem for

$$
\partial_{t} U+\partial_{x} F(U)=0
$$

is the Cauchy problem with Riemann initial data

$$
U^{0}(x)= \begin{cases}U_{l} & \text { if } x<0, \\ U_{r} & \text { if } x>0 .\end{cases}
$$

Since this initial data satisfies $U^{0}(\lambda x)=U^{0}(x)$ for any $\lambda>0$, one checks easily by homogeneity that one can expect a solution to (3.1) of the form

$$
U(t, x)=R_{e x}\left(x / t, U_{l}, U_{r}\right),
$$

with $R_{e x}$ the exact Riemann solver. 2).

The solution to the Riemann problem depends only on $x / t$, thus is constant on the lines $x / t=c s t$ (see Figure

\subsection{Approximate Riemann solvers}

Definition 3.1. An approximate Riemann solver for (3.1) is a vector function $R\left(x / t, U_{l}, U_{r}\right)$ that is an approximation of the solution to the Riemann problem, in the sense that it must satisfy the consistency relation

$$
R(x / t, U, U)=U,
$$

and the conservativity identity

$$
F_{l}\left(U_{l}, U_{r}\right)=F_{r}\left(U_{l}, U_{r}\right),
$$

where the left and right numerical fluxes are defined by

$$
\begin{aligned}
& F_{l}\left(U_{l}, U_{r}\right)=F\left(U_{l}\right)-\int_{-\infty}^{0}\left(R\left(v, U_{l}, U_{r}\right)-U_{l}\right) d v, \\
& F_{r}\left(U_{l}, U_{r}\right)=F\left(U_{r}\right)+\int_{0}^{\infty}\left(R\left(v, U_{l}, U_{r}\right)-U_{r}\right) d v .
\end{aligned}
$$




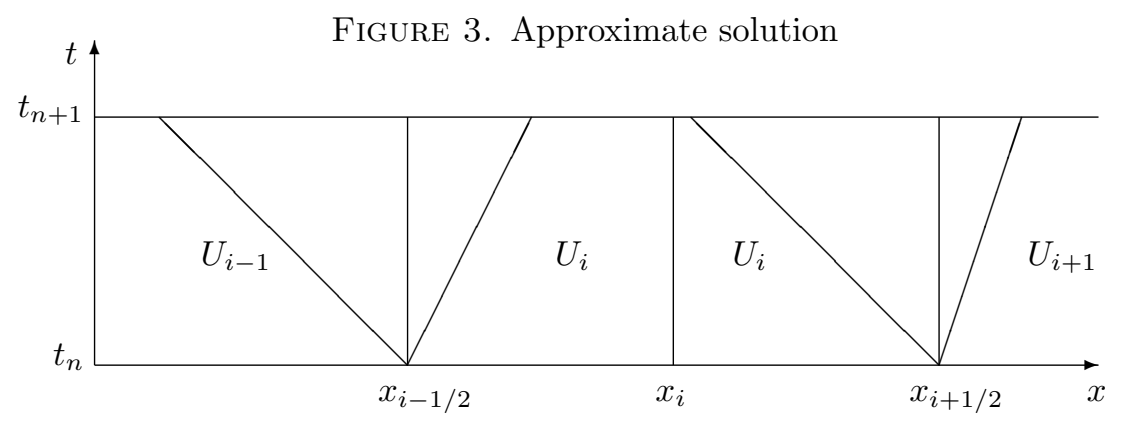

The approximate Riemann solver is called dissipative with respect to a convex entropy $\eta$ for (3.1) if

$$
G_{r}\left(U_{l}, U_{r}\right)-G_{l}\left(U_{l}, U_{r}\right) \leq 0
$$

where

$$
\begin{aligned}
& G_{l}\left(U_{l}, U_{r}\right)=G\left(U_{l}\right)-\int_{-\infty}^{0}\left(\eta\left(R\left(v, U_{l}, U_{r}\right)\right)-\eta\left(U_{l}\right)\right) d v \\
& G_{r}\left(U_{l}, U_{r}\right)=G\left(U_{r}\right)+\int_{0}^{\infty}\left(\eta\left(R\left(v, U_{l}, U_{r}\right)\right)-\eta\left(U_{r}\right)\right) d v
\end{aligned}
$$

and $G$ is the entropy flux associated to $\eta, \quad G^{\prime}=\eta^{\prime} F^{\prime}$.

To an approximate Riemann solver we can associate a consistent conservative scheme, as follows.

\subsection{Interpretation of an approximate solver}

Take $U^{n}(x)$ piecewise constant over the mesh with value $U_{i}^{n}$ in each cell $C_{i}$. We can consider that close to each interface point $x_{i+1 / 2}$, we have to solve a translated Riemann problem. Since (3.1) is invariant under translation in time and space, we can think of sticking together the local approximate Riemann solutions $R\left(\left(x-x_{i+1 / 2}\right) /\left(t-t_{n}\right), U_{i}^{n}, U_{i+1}^{n}\right)$, at least for times such that these solutions do not interact (see Figure 3 ).

There is no interaction until time $t_{n+1}$ under a CFL condition $1 / 2$, in the sense that

$$
\begin{aligned}
& x / t<-\frac{\Delta x_{i}}{2 \Delta t} \quad \Rightarrow \quad R\left(x / t, U_{i}, U_{i+1}\right)=U_{i} \\
& x / t>\frac{\Delta x_{i+1}}{2 \Delta t} \quad \Rightarrow \quad R\left(x / t, U_{i}, U_{i+1}\right)=U_{i+1}
\end{aligned}
$$

Thus we define an approximate solution $U(t, x)$ for $t_{n} \leq t<t_{n+1}$ by

$$
U(t, x)=R\left(\frac{x-x_{i+1 / 2}}{t-t_{n}}, U_{i}^{n}, U_{i+1}^{n}\right) \quad \text { if } x_{i}<x<x_{i+1}
$$


Then, we define $U_{i}^{n+1}$ to be the average over $C_{i}$ of this approximate solution at time $t_{n+1}-0$. According to the definition of $F_{l}$ and $F_{r}$ and by using the CFL condition, we get

$$
\begin{aligned}
U_{i}^{n+1} & =\frac{1}{\Delta x_{i}} \int_{x_{i-1 / 2}}^{x_{i+1 / 2}} U\left(t_{n+1}-0, x\right) d x \\
& =\frac{1}{\Delta x_{i}} \int_{0}^{\Delta x_{i} / 2} R\left(x / \Delta t, U_{i-1}^{n}, U_{i}^{n}\right) d x+\frac{1}{\Delta x_{i}} \int_{-\Delta x_{i} / 2}^{0} R\left(x / \Delta t, U_{i}^{n}, U_{i+1}^{n}\right) d x \\
& =U_{i}^{n}+\frac{1}{\Delta x_{i}} \int_{0}^{\Delta x_{i} / 2}\left(R\left(x / \Delta t, U_{i-1}^{n}, U_{i}^{n}\right)-U_{i}^{n}\right) d x \\
& \quad+\frac{1}{\Delta x_{i}} \int_{-\Delta x_{i} / 2}^{0}\left(R\left(x / \Delta t, U_{i}^{n}, U_{i+1}^{n}\right)-U_{i}^{n}\right) d x \\
& =U_{i}^{n}-\frac{\Delta t}{\Delta x_{i}}\left[F_{l}\left(U_{i}^{n}, U_{i+1}^{n}\right)-F_{r}\left(U_{i-1}^{n}, U_{i}^{n}\right)\right] .
\end{aligned}
$$

Therefore with the conservativity and consistency assumptions (3.4), (3.5), this is a consistent conservative scheme, with numerical flux $F\left(U_{l}, U_{r}\right)=F_{l}\left(U_{l}, U_{r}\right)=F_{r}\left(U_{l}, U_{r}\right)$.

\subsection{Entropy}

If the approximate Riemann solver is dissipative with respect to a convex entropy $\eta$, then a discrete entropy inequality holds according to

$$
\begin{aligned}
\eta\left(U_{i}^{n+1}\right) \leq & \frac{1}{\Delta x_{i}} \int_{0}^{\Delta x_{i} / 2} \eta\left(R\left(x / \Delta t, U_{i-1}^{n}, U_{i}^{n}\right)\right) d x \\
& \quad+\frac{1}{\Delta x_{i}} \int_{-\Delta x_{i} / 2}^{0} \eta\left(R\left(x / \Delta t, U_{i}^{n}, U_{i+1}^{n}\right)\right) d x \\
= & \eta\left(U_{i}^{n}\right)-\frac{\Delta t}{\Delta x_{i}}\left[G_{l}\left(U_{i}^{n}, U_{i+1}^{n}\right)-G_{r}\left(U_{i-1}^{n}, U_{i}^{n}\right)\right] .
\end{aligned}
$$

Under the entropy assumption $G_{r} \leq G_{l}$ this gives

$$
\eta\left(U_{i}^{n+1}\right)-\eta\left(U_{i}^{n}\right)+\frac{\Delta t}{\Delta x_{i}}\left[G\left(U_{i}^{n}, U_{i+1}^{n}\right)-G\left(U_{i-1}^{n}, U_{i}^{n}\right)\right] \leq 0
$$

for any numerical entropy flux function $G\left(U_{l}, U_{r}\right)$ such that $G_{r}\left(U_{l}, U_{r}\right) \leq G\left(U_{l}, U_{r}\right) \leq G_{l}\left(U_{l}, U_{r}\right)$.

\subsection{Invariant domains}

The invariant domains are also analyzed easily for approximate Riemann solvers.

Proposition 3.2. Assume that $R$ is an approximate Riemann solver that preserves a convex invariant domain $\mathcal{U}$, in the sense that

$$
U_{l}, U_{r} \in \mathcal{U} \Rightarrow R\left(x / t, U_{l}, U_{r}\right) \in \mathcal{U} \text { for any value of } x / t
$$

Then the numerical scheme associated to $R$ also preserves $\mathcal{U}$.

This is easily seen by the convex averaging formula

$$
U_{i}^{n+1}=\frac{1}{\Delta x_{i}} \int_{x_{i-1 / 2}}^{x_{i+1 / 2}} U\left(t_{n+1}-0, x\right) d x
$$


Figure 4. A simple solver

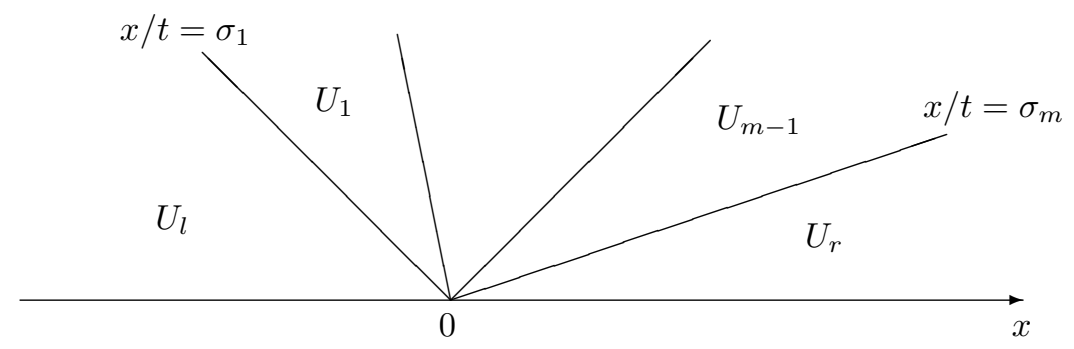

where $U\left(t_{n+1}-0, x\right)$ is obtained by sticking the local solvers

$$
R\left(\frac{x-x_{i+1 / 2}}{t-t_{n}}, U_{i}, U_{i+1}\right)
$$

\subsection{Simple solvers}

A simple solver is an approximate Riemann solver consisting in a set of finitely many simple discontinuities. This means that there exists a finite number $m \geq 1$ of speeds $\sigma_{0}=-\infty<\sigma_{1}<\cdots<\sigma_{m}<\sigma_{m+1}=+\infty$, and intermediate states $U_{0}=U_{l}, U_{1}, \ldots, U_{m-1}, U_{m}=U_{r}$ (depending on $U_{l}$ and $U_{r}$ ), such that as shown on Figure 4 ,

$$
R\left(x / t, U_{l}, U_{r}\right)=U_{k} \quad \text { if } \quad \sigma_{k}<x / t<\sigma_{k+1} .
$$

Then the conservativity identity becomes

$$
\sum_{k=1}^{m} \sigma_{k}\left(U_{k}-U_{k-1}\right)=F\left(U_{r}\right)-F\left(U_{l}\right)
$$

and the entropy inequality becomes

$$
\sum_{k=1}^{m} \sigma_{k}\left(\eta\left(U_{k}\right)-\eta\left(U_{k-1}\right)\right) \geq G\left(U_{r}\right)-G\left(U_{l}\right)
$$

Conservativity thus enables to define the intermediate fluxes $F_{k}, k=0, \ldots, m$, by

$$
F_{k}-F_{k-1}=\sigma_{k}\left(U_{k}-U_{k-1}\right), \quad F_{0}=F\left(U_{l}\right), F_{m}=F\left(U_{r}\right),
$$

which is a kind of generalization of the Rankine-Hugoniot relation. The numerical flux is then given by

$$
F\left(U_{l}, U_{r}\right)=F_{k}, \quad \text { where } k \text { is such that } \sigma_{k} \leq 0 \leq \sigma_{k+1},
$$

or equivalently by

$$
\begin{aligned}
F\left(U_{l}, U_{r}\right) & =F\left(U_{l}\right)+\sum_{\sigma_{k}<0} \sigma_{k}\left(U_{k}-U_{k-1}\right) \\
& =F\left(U_{r}\right)-\sum_{\sigma_{k}>0} \sigma_{k}\left(U_{k}-U_{k-1}\right) .
\end{aligned}
$$




\subsection{HLL solver}

The most simple approximate Riemann solver is the well-known HLL solver,

$$
R\left(x / t, U_{l}, U_{r}\right)=\left\{\begin{array}{l}
U_{l} \text { if } x / t<c_{1}, \\
\frac{c_{2} U_{r}-F\left(U_{r}\right)}{c_{2}-c_{1}}+\frac{-c_{1} U_{l}+F\left(U_{l}\right)}{c_{2}-c_{1}} \\
U_{r} \text { if } c_{2}<x / t,
\end{array} \quad \text { if } c_{1}<x / t<c_{2},\right.
$$

where $c_{1}<c_{2}$ are two parameters. The HLL numerical flux is given by

$$
F\left(U_{l}, U_{r}\right)=\left\{\begin{array}{l}
F\left(U_{l}\right) \text { if } 0<c_{1}, \\
\frac{c_{2} F\left(U_{l}\right)-c_{1} F\left(U_{r}\right)}{c_{2}-c_{1}}+\frac{c_{1} c_{2}}{c_{2}-c_{1}}\left(U_{r}-U_{l}\right) \quad \text { if } c_{1}<0<c_{2}, \\
F\left(U_{r}\right) \text { if } c_{2}<0 .
\end{array}\right.
$$

- In case $c_{2}=-c_{1}$ one gets the Lax-Friedrichs scheme.

- In case $c_{1}>0$ one gets the upwind scheme $F\left(U_{l}, U_{r}\right)=F\left(U_{l}\right)$.

Properties:

- One can prove entropy inequalities and invariant domains properties if $c_{1} \leq \lambda_{j}(U) \leq c_{2}$, where $\lambda_{j}(U)$ are the eigenvalues of $F^{\prime}(U)$.

The choice for the best accuracy is

$$
c_{1}=\inf _{U=U_{l}, U_{r}} \inf _{j} \lambda_{j}(U), \quad c_{2}=\sup _{U=U_{l}, U_{r}} \sup _{j} \lambda_{j}(U) .
$$

- The HLL solver works for arbitrary nonlinearity $F$

- It well resolves the waves with maximal and minimal speeds $\lambda_{1}(U)$ and $\lambda_{p}(U)$, but puts a lot of diffusion on intermediate waves.

Generalization with three waves for gas dynamics: HLLC scheme [1]. All the waves are well-resolved, and

- Density and internal energy remain nonnegative,

- Entropy inequalities hold.

More generally, approximate Riemann solvers can be built via relaxation approximations, see [1].

\section{SECOND-ORDER SCHEMES}

\subsection{Reconstruction operator}

A classical method to go to second-order accuracy: use first-order fluxes and a reconstruction operator.

Definition 4.1. A second-order reconstruction is an operator which to a sequence $U_{i}$ associates values $U_{i+1 / 2-}$, $U_{i+1 / 2+}$ for $i \in \mathbb{Z}$, in such a way that it is conservative,

$$
\frac{U_{i-1 / 2+}+U_{i+1 / 2-}}{2}=U_{i}
$$

and it is second-order in the sense that whenever for all $i$,

$$
U_{i}=\frac{1}{\Delta x_{i}} \int_{C_{i}} U(x) d x
$$


for some smooth function $U(x)$, then

$$
U_{i+1 / 2-}=U\left(x_{i+1 / 2}\right)+O\left(h^{2}\right), \quad U_{i+1 / 2+}=U\left(x_{i+1 / 2}\right)+O\left(h^{2}\right) .
$$

The second-order scheme is then defined as

$$
\begin{gathered}
U_{i}^{n+1}-U_{i}^{n}+\frac{\Delta t}{\Delta x_{i}}\left(F_{i+1 / 2}-F_{i-1 / 2}\right)=0, \\
F_{i+1 / 2}=F\left(U_{i+1 / 2-}^{n}, U_{i+1 / 2+}^{n}\right),
\end{gathered}
$$

where $F\left(U_{l}, U_{r}\right)$ is a given consistent numerical flux, and where $\left(U_{i+1 / 2 \pm}^{n}\right)_{i \in \mathbb{Z}}$ are obtained from the reconstruction of $\left(U_{i}^{n}\right)_{i \in \mathbb{Z}}$.

\subsection{Accuracy}

Proposition 4.2 (Second-order accuracy). Assume that the numerical flux is Lipschitz continuous and consistent. If for all $i$,

$$
U_{i}^{n}=\frac{1}{\Delta x_{i}} \int_{C_{i}} U\left(t_{n}, x\right) d x
$$

for some smooth solution $U(t, x)$ to (3.1), then $U_{i}^{n+1}$ satisfies for all $i$

$$
U_{i}^{n+1}=\frac{1}{\Delta x_{i}} \int_{C_{i}} U\left(t_{n+1}, x\right) d x+\Delta t\left(\frac{1}{\Delta x_{i}}\left(\mathcal{F}_{i+1 / 2}-\mathcal{F}_{i-1 / 2}\right)\right),
$$

where

as $\Delta t$ and $h=\sup _{i} \Delta x_{i}$ tend to 0.

$$
\mathcal{F}_{i+1 / 2}=O(\Delta t)+O\left(h^{2}\right)
$$

The proof is given in [1].

\subsection{Stability}

The reconstruction is said to preserve a convex invariant domain $\mathcal{U}$ if

$$
U_{i} \in \mathcal{U} \text { for all } i \Rightarrow U_{i+1 / 2 \pm} \in \mathcal{U} \text { for all } i
$$

Proposition 4.3. If under a CFL condition the numerical flux preserves a convex invariant domain $\mathcal{U}$, and if the reconstruction also preserves this invariant domain, then under the half original CFL condition, the second-order scheme also preserves this invariant domain.

The proof is given in [1] and relies on an interpretation of the scheme via a piecewise constant function by half cell (see Figure 5).

\subsection{Examples of reconstruction operators}

The second-order reconstruction must also be nonoscillatory in some sense, see [2], [3].

- The minmod reconstruction for scalar functions is defined as

$$
U_{i-1 / 2+}=U_{i}-\frac{\Delta x_{i}}{2} D U_{i}, \quad U_{i+1 / 2-}=U_{i}+\frac{\Delta x_{i}}{2} D U_{i}
$$


FIGURE 5. Interpretation of second-order scheme via half cell values

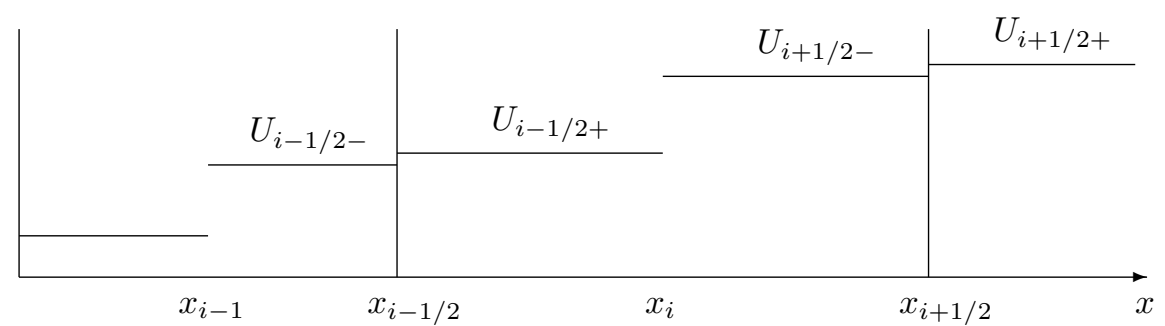

with

and

$$
D U_{i}=\operatorname{minmod}\left(\frac{U_{i}-U_{i-1}}{\left(\Delta x_{i-1}+\Delta x_{i}\right) / 2}, \frac{U_{i+1}-U_{i}}{\left(\Delta x_{i}+\Delta x_{i+1}\right) / 2}\right),
$$

$$
\operatorname{minmod}(x, y)=\left\{\begin{array}{l}
\min (x, y) \text { if } x, y \geq 0, \\
\max (x, y) \text { if } x, y \leq 0, \\
0 \text { otherwise }
\end{array}\right.
$$

- For the gas dynamics system, the conservative variable is $U \equiv\left(\rho, \rho u, \rho u^{2} / 2+\rho e\right)$. Given the values $U_{i}$, we would like to find second-order accurate values $U_{i+1 / 2 \pm}$ such that

$$
\frac{U_{i-1 / 2+}+U_{i+1 / 2-}}{2}=U_{i}
$$

Knowing that the $U_{i}$ have positive densities and positive internal energies $\rho_{i}>0, e_{i}>0$, we require the same properties for the reconstructed values, $\rho_{i+1 / 2 \pm}>0, e_{i+1 / 2 \pm}>0$.

The first step of the construction is to write a parametrization of all possible values $U_{i-1 / 2+}, U_{i+1 / 2-}$ satisfying (4.13). An algebraic computation gives that they must be of the form

$$
\begin{gathered}
\rho_{i-1 / 2+}=\rho_{i}-\frac{\Delta x_{i}}{2} D \rho_{i}, \quad \rho_{i+1 / 2-}=\rho_{i}+\frac{\Delta x_{i}}{2} D \rho_{i} \\
u_{i-1 / 2+}=u_{i}-\frac{\rho_{i+1 / 2-}}{\rho_{i}} \frac{\Delta x_{i}}{2} D u_{i}, \quad u_{i+1 / 2-}=u_{i}+\frac{\rho_{i-1 / 2+}}{\rho_{i}} \frac{\Delta x_{i}}{2} D u_{i} \\
\rho_{i-1 / 2+} e_{i-1 / 2+}=\rho_{i} \widetilde{e}_{i}-\frac{\Delta x_{i}}{2} D(\rho e)_{i}, \quad \rho_{i+1 / 2-} e_{i+1 / 2-}=\rho_{i} \widetilde{e}_{i}+\frac{\Delta x_{i}}{2} D(\rho e)_{i} \\
\text { with } \quad \widetilde{e}_{i}=e_{i}-\frac{\rho_{i-1 / 2+} \rho_{i+1 / 2-}}{\rho_{i}^{2}} \frac{\Delta x_{i}^{2}}{8} D u_{i}^{2} .
\end{gathered}
$$

We observe then that the second-order accuracy means that $D \rho_{i}, D u_{i}, D(\rho e)_{i}$ must be consistent with the derivatives of $\rho, u, \rho e$ respectively. Concerning the positivity conditions, they can be expressed as

$$
\frac{\Delta x_{i}}{2}\left|D \rho_{i}\right|<\rho_{i}, \quad \frac{\Delta x_{i}^{2}}{8} D u_{i}^{2}<\frac{\rho_{i}}{\rho_{i-1 / 2+} \rho_{i+1 / 2-}}\left(\rho_{i} e_{i}-\frac{\Delta x_{i}}{2}\left|D(\rho e)_{i}\right|\right) .
$$

Therefore, the computation of the slopes can be done as follows: we first compute $D \rho_{i}, D u_{i}, D(\rho e)_{i}$ by the minmod slope formula, where the $U_{i}$ are replaced by $\rho_{i}, u_{i}, \rho_{i} e_{i}$ respectively, and then we eventually diminish the absolute value of $D u_{i}$ so as to satisfy the second inequality in (4.15). This computation gives consistent values of $D \rho_{i}, D u_{i}, D(\rho e)_{i}$.

Thus the reconstruction is second-order accurate, and preserves the nonnegativity of density and internal energy. 


\subsection{Second-order accuracy in time}

The second-order accuracy in time is usually recovered by the Heun method, which reads as follows. The second-order method in $x$ can be written as

$$
U^{n+1}=U^{n}+\Delta t \Phi\left(U^{n}\right)
$$

where $U=\left(U_{i}\right)_{i \in \mathbb{Z}}$, and $\Phi$ is a nonlinear operator depending on the mesh. Then the second-order scheme in time and space is

$$
\begin{aligned}
\widetilde{U}^{n+1} & =U^{n}+\Delta t \Phi\left(U^{n}\right), \\
\widetilde{U}^{n+2} & =\widetilde{U}^{n+1}+\Delta t \Phi\left(\widetilde{U}^{n+1}\right), \\
U^{n+1} & =\frac{U^{n}+\widetilde{U}^{n+2}}{2} .
\end{aligned}
$$

It is easy to see that if the numerical flux does not depend explicitly on $\Delta t$, this procedure gives a fully secondorder scheme in the sense that we get an error in $O\left(\Delta t^{2}\right)+O\left(h^{2}\right)$. The invariant domains are also preserved because of the average, without any further limitation on the CFL.

\subsection{Test 1: Burgers equation}

The first numerical illustration we propose is for Burgers equation

$$
\partial_{t} U+\partial_{x}\left(U^{2} / 2\right)=0
$$

with $U \in \mathbb{R}$. The exact solution, in continuous line on figure, contains a shock and a rarefaction. The numerical solutions obtained with the HLL scheme at first and second-order are shown.

\section{Burgers equation}

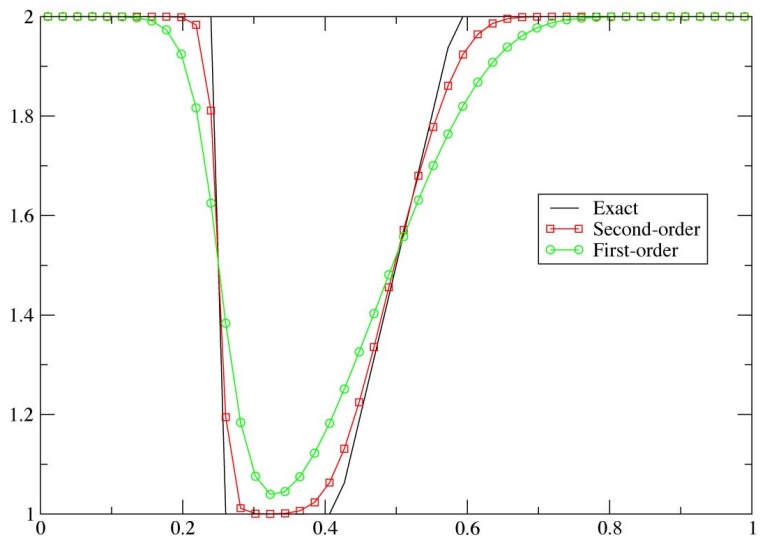

\subsection{Test 2: Gas dynamics}

The second numerical illustration is on the gas dyanmics system (1.4). It is for a Riemann problem with a shock, a contact discontinuity and a rarefaction wave. First and second-order computations obtained with the HLLC scheme are shown on figures. 


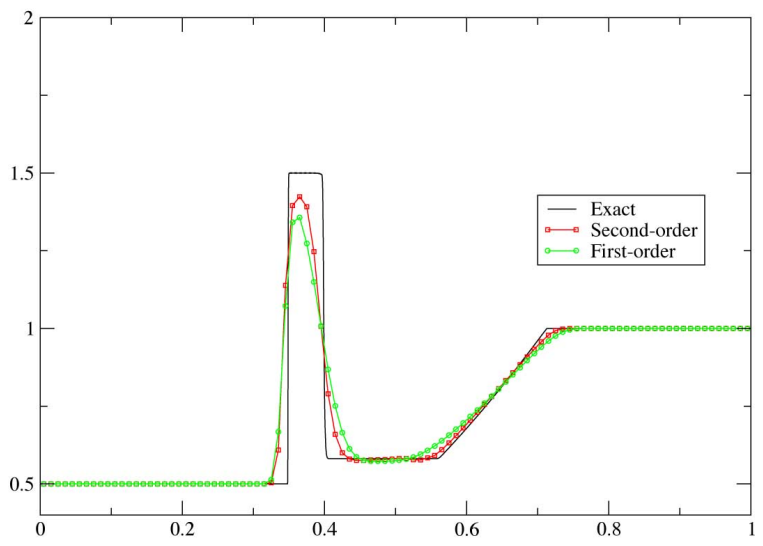

Gas dynamics, Velocity

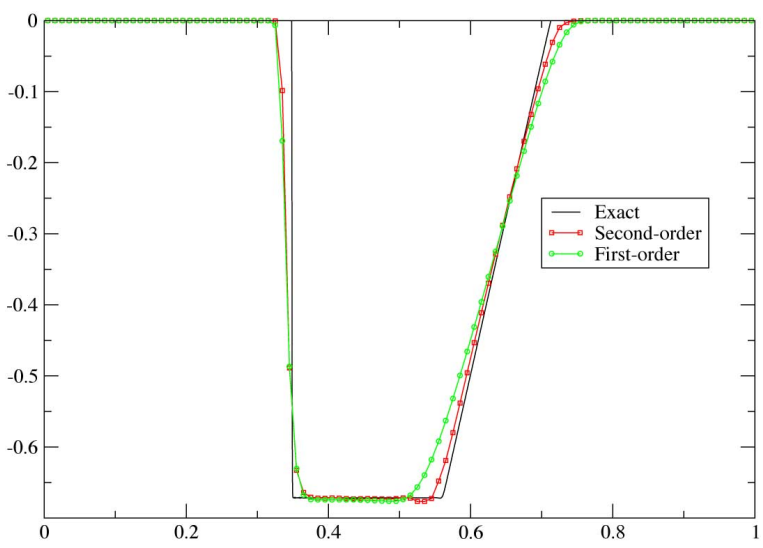

Gas dynamics, Internal energy

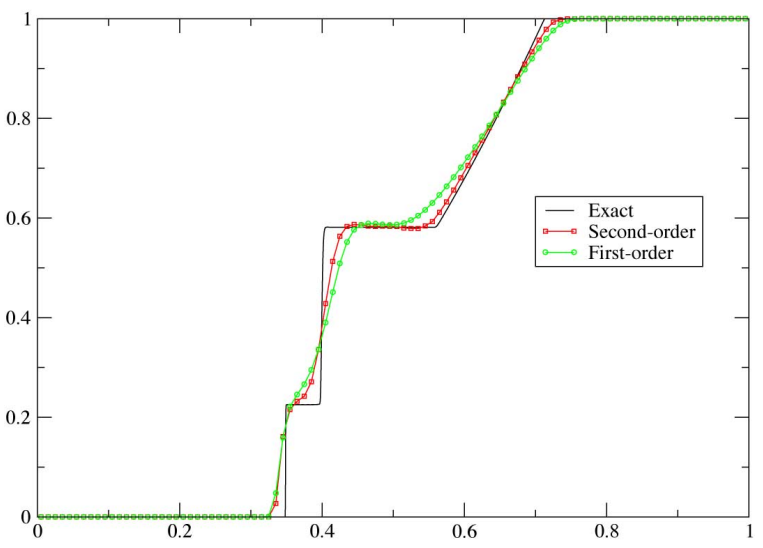

5. Multidimensional problems

\subsection{Bidimensional systems of conservation laws}

Bidimensional systems of conservation laws are of the form

$$
\partial_{t} U+\partial_{1}\left(F_{1}(U)\right)+\partial_{2}\left(F_{2}(U)\right)=0,
$$


Figure 6 . Two-dimensional setting

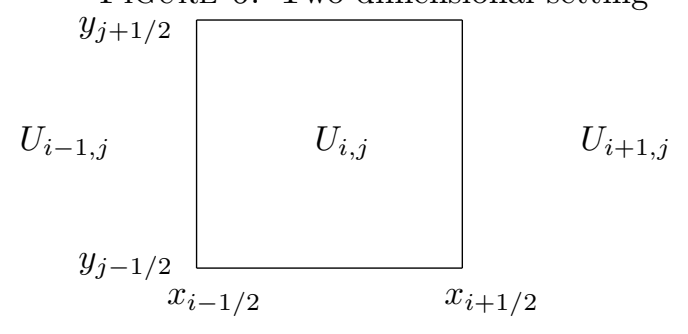

where the space variable is $x=\left(x_{1}, x_{2}\right) \in \mathbb{R}^{2}$, and the partial derivatives $\partial_{1}, \partial_{2}$ refer to these variables. The unknown is $U(t, x) \in \mathbb{R}^{p}$. More generally for a two-dimensional quasilinear system

$$
\partial_{t} U+A_{1}(U) \partial_{1} U+A_{2}(U) \partial_{2} U=0
$$

we can consider planar solutions, i.e. solutions of the form $U(t, x)=U(t, \zeta)$ with $\zeta=x \cdot n$ and $n=\left(n^{1}, n^{2}\right)$ is any unit vector in $\mathbb{R}^{2}$, which leads to

$$
\partial_{t} U+A_{n}(U) \partial_{\zeta} U=0
$$

with

$$
A_{n}(U)=n^{1} A_{1}(U)+n^{2} A_{2}(U) .
$$

The notions introduced previously can be applied to the one-dimensional quasilinear system (5.3), and by this way one defines hyperbolicity, entropies, and other notions for (5.2) by requiring the one-dimensional properties for all directions $n$. In particular, the system (5.2) is hyperbolic if for any unit vector $n$ in $\mathbb{R}^{2}$ and any $U, A n(U)$ is diagonalizable.

\subsection{Schemes by interface}

Let us consider a two-dimensional system of conservation laws

$$
\partial_{t} U+\partial_{1}\left(F_{1}(U)\right)+\partial_{2}\left(F_{2}(U)\right)=0
$$

and a two-dimensional cartesian mesh made of cells

$$
C_{i j}=\left(x_{i-1 / 2}, x_{i+1 / 2}\right) \times\left(y_{j-1 / 2}, y_{j+1 / 2}\right)
$$

with lengths $\Delta x_{i}=x_{i+1 / 2}-x_{i-1 / 2}, \Delta y_{j}=y_{j+1 / 2}-y_{j-1 / 2}$. The finite volume formula by interface then reads

$$
U_{i j}^{n+1}-U_{i j}^{n}+\frac{\Delta t}{\Delta x_{i}}\left(F_{i+1 / 2, j}^{1}-F_{i-1 / 2, j}^{1}\right)+\frac{\Delta t}{\Delta y_{j}}\left(F_{i, j+1 / 2}^{2}-F_{i, j-1 / 2}^{2}\right)=0 .
$$

The interface fluxes are computed as in one dimension

$$
F_{i+1 / 2, j}^{1}=F^{1}\left(U_{i, j}, U_{i+1, j}\right), \quad F_{i, j+1 / 2}^{2}=F^{2}\left(U_{i, j}, U_{i, j+1}\right),
$$

where $F^{1}\left(U_{l}, U_{r}\right), F^{2}\left(U_{l}, U_{r}\right)$ are one-dimensional numerical fluxes corresponding to the nonlinearities $F^{1}(U)$ and $F^{2}(U)$ (see Figure 6).

Proposition 5.1. If the one-dimensional fluxes let invariant a domain $\mathcal{U}$ under a CFL condition

$$
\Delta t a\left(U_{i}, U_{i+1}\right) \leq \Delta x_{i},
$$


FiguRE 7 . Reconstruction by line

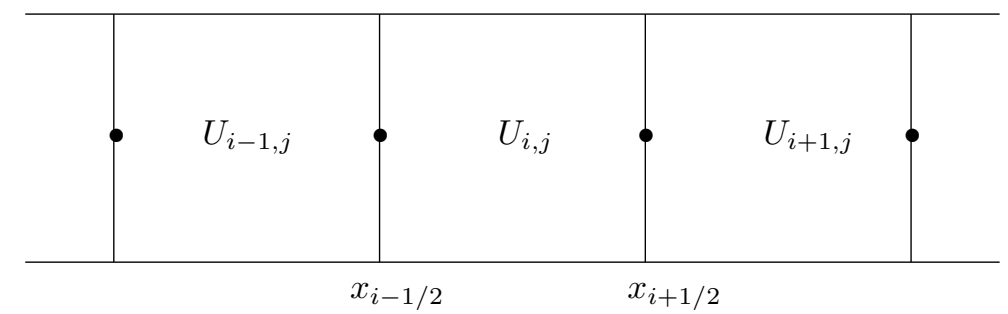

then the two-dimensional scheme also lets this domain invariant, under the half CFL condition

$$
\Delta t a\left(U_{i, j}, U_{i+1, j}\right) \leq \frac{1}{2} \Delta x_{i}, \quad \Delta t a\left(U_{i, j}, U_{i, j+1}\right) \leq \frac{1}{2} \Delta y_{j}
$$

A similar result is true for entropy inequalities.

\subsection{Second-order accuracy}

The reconstruction method extends easily to several dimensions. The interface fluxes are computed at reconstructed values

$$
F_{i+1 / 2, j}^{1}=F^{1}\left(U_{i+1 / 2-, j}, U_{i+1 / 2+, j}\right), \quad F_{i, j+1 / 2}^{2}=F^{2}\left(U_{i, j+1 / 2-}, U_{i, j+1 / 2+}\right),
$$

where $\left(U_{i+1 / 2 \pm, j}\right)_{i \in \mathbb{Z}}$ is obtained by a one-dimensional reconstruction of $\left(U_{i, j}\right)_{i \in \mathbb{Z}}$ at fixed $j$ (reconstruction by line, see Figure 7$)$, and $\left(U_{i, j+1 / 2 \pm}\right)_{j \in \mathbb{Z}}$ is obtained by a one-dimensional reconstruction of $\left(U_{i, j}\right)_{j \in \mathbb{Z}}$ at fixed $i$ (reconstruction by column).

Proposition 5.2. If the one-dimensional fluxes let invariant a domain $\mathcal{U}$ under a CFL condition

$$
\Delta t a\left(U_{i}, U_{i+1}\right) \leq \Delta x_{i}
$$

and if the reconstruction also lets $\mathcal{U}$ invariant, then the two-dimensional second-order scheme also lets this domain invariant, under the $1 / 4$ CFL condition

$$
\Delta t a\left(U_{i, j}, U_{i+1, j}\right) \leq \frac{1}{4} \Delta x_{i}, \quad \Delta t a\left(U_{i, j}, U_{i, j+1}\right) \leq \frac{1}{4} \Delta y_{j}
$$

\subsection{Test 3: Gas dynamics, random velocity}

This test case for gas dynamics in two dimensions is intended to asses the stability of the schemes. The initial data has constant density, vanishing internal energy, and randomly distributed velocity. The solution quickly develops small vacuum areas everywhere, and then kinetic energy is transfered to potential energy. We use the HLLC scheme at second order, which has the property to ensure proper computation of vacuum, see [1]. The energies with respect to time are plotted on figure. 
2d Gas dynamics

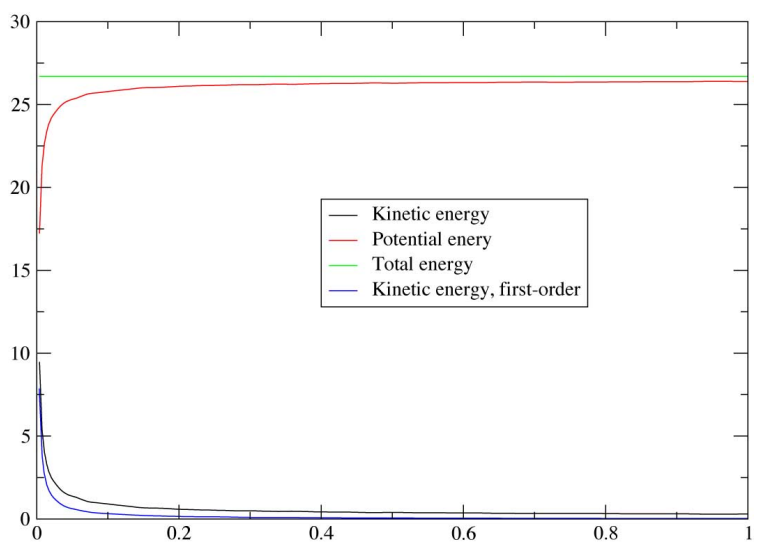

\section{BIBLIOGRAPHY}

[1] F. Bouchut, Nonlinear stability of finite volume methods for hyperbolic conservation laws, and well-balanced schemes for sources, Frontiers in Mathematics series, Birkhäuser, 2004.

[2] R. Eymard, T. Gallouët, R. Herbin, Finite volume methods, In Handbook of Numerical Analysis (Vol. VII), P.G. Ciarlet and J.-L. Lions editors, North-Holland, 2000.

[3] E. Godlewski, P.-A. Raviart, Hyperbolic systems of conservation laws, Mathématiques \& Applications 3/4, Ellipses, Paris, 1991.

[4] E. Godlewski, P.-A. Raviart, Numerical approximation of hyperbolic systems of conservation laws, Applied Mathematical Sciences, 118, Springer-Verlag, New York, 1996.

[5] R.J. LeVeque, Finite volume methods for hyperbolic problems, Cambridge Univ. Press, 2002.

[6] D. Serre, Systems of conservation laws. 1. Hyperbolicity, entropies, shock waves, Translated from the 1996 French original by I. N. Sneddon, Cambridge University Press, Cambridge, 1999.

[7] E.F. Toro, Riemann solvers and numerical methods for fluid dynamics: a practical introduction, second edition, Springer-Verlag, 1999.

Most of the material in this presentation can be found in more details in reference [1]. The motivation here is to give basic tools for solving generic systems of conservation laws. 